\title{
A case report of wrist synovial infection due to Mycobacterium jacuzzii, Iran
}

\author{
Fatemeh Sakhaee ${ }^{1}$, Morteza Masoumi ${ }^{1}$, Farzam Vaziri ${ }^{1,2}$, Seyed Davar Siadat ${ }^{1,2}$ and Abolfazl Fateh ${ }^{1,2^{*}}$ (D)
}

\begin{abstract}
Background: Mycobacterium jacuzzii (M. jacuzzii) was first isolated in 2003 by insertion of breast implants in Tel Aviv, Israel. In this case report, we describe our experience in detection of M. jacuzzii using phenotypic and genotypic test of wrist synovial sample.

Case presentation: A 73-year-old woman complained of pain and swelling in the right wrist for 4 months. Her body temperature was $37-38^{\circ} \mathrm{C}$, and symptoms, such as pain, swelling, and some movement limitation, were reported. Clinical laboratory parameters showed an elevated C-reactive protein (CRP) level, erythrocyte sedimentation rate (ESR), and white blood cells (WBC) count. The sequences of hsp65, rpoB, 165 rDNA, and sodA genes indicated very high homology to M. jacuzzii.

Conclusion: We report a case of synovial infection caused by M. jacuzzii in a patient with severe wrist pain in Iran, who was treated with amikacin, levofloxacin, and ethambutol. The outcomes of treatment after 8 months were positive, and no recurrence of infection was reported in the patient.
\end{abstract}

Keywords: Mycobacterium jacuzzii, Synovial infection, Iran

\section{Background}

In the past several years, the outbreak of human diseases associated with nontuberculous mycobacteria (NTM) has been increasing. The cause of this increase is probably multifactorial, depending on the host, nature of infectious agent, and their interactions [1]. Due to the redundancy of tissue and synovial fluid and a higher probability of penetrating injury, NTM tenosynovitis happens most frequently in the hand and wrist. Usually, slowly growing mycobacteria species, especially Mycobacterium (M.) marinum, are involved [2]. In the present study, we report for the first time the isolation of $M$. jacuzzii from wrist synovial in a 73-year-old woman patient.

\footnotetext{
* Correspondence: afateh2@gmail.com

'Department of Mycobacteriology and Pulmonary Research, Pasteur Institute of Iran, Tehran, Iran

${ }^{2}$ Microbiology Research Center (MRC), Pasteur Institute of Iran, Tehran, Iran
}

\section{Case presentation}

A 73-year-old woman complained of pain and swelling in the right wrist for 4 months. Her body temperature was $37-38^{\circ} \mathrm{C}$, and symptoms, such as pain, swelling, and some movement limitation, were reported. Moreover, clinical laboratory parameters showed an elevated C-reactive protein (CRP) level $(24 \mathrm{mg} / \mathrm{L})$, erythrocyte sedimentation rate (ESR) $(51 \mathrm{~mm} / \mathrm{h}$ ), and white blood cells (WBC) count (121,000 cells per cubic millimeter). Also, the rheumatoid factor and antinuclear antibody were negative. No history of trauma, diabetes, or immunosuppressive drug consumption was reported. Meanwhile, the patient received a local injection of methylprednisolone $(20 \mathrm{mg}$ daily/2 weeks). His swollen partially resolved but worsened again.

The synovial fluid sample was aspirated from the wrist and sent to Pasteur Institute of Iran in January 2019 for evaluating the presence of Mycobacterium (M.) tuberculosis. The results of smear test were positive for acid-fast bacillus (AFB). 
A needle biopsy was performed for pathological and microbiological tests. After tissue evaluation, the synovium, containing epithelioid cells, fibrotic changes, and numerous noncaseating granulomas, was obviously thickened due to chronic inflammation. Ziehl-Neelsen staining indicated AFB in the tissue.

A synovial fluid sample was cultured on the LowensteinJensen medium. After 5 days, the results indicated rapidlygrowing mycobacteria (RGM) with smooth, small, and non-pigmented colonies. The results of biochemical tests were negative for three-day arylsulfatase growth at $45^{\circ} \mathrm{C}$, niacin accumulation, and nitrate reductase, while they were positive for growth on MacConkey agar without crystal violet, heat-resistant catalase, and iron uptake tests.

The multilocus sequence analysis was also performed using partial $h s p 65, \operatorname{rpoB}$, sodA, and full $16 S \mathrm{rDNA}$ genes, as previously described [2-4]. The results of biochemical tests indicated homology to $M$. wolinskyi, while the sequences of $h s p 65$ (441-bp), rрoB (750-bp), $16 S$ rDNA ( 1500-bp), and sodA (524-bp) genes indicated very high homology to M. jacuzzii (Fig. 1), as previously shown [5].

The drug susceptibility pattern (DSP) test was performed according to the Clinical and Laboratory
Standards Institute (CLSI) guidelines [6]. The results indicated that the $M$. jacuzzii was extremely resistant to isoniazid, rifampin, clarithromycin, sulfamethoxazoletrimethoprim, capreomycin, cycloserine, tobramycin, cefoxitin, imipenem and streptomycin and highly susceptible to amikacin, levofloxacin, doxycycline, ofloxacin, ethambutol, and ciprofloxacin.

Antimicrobial therapy was performed according to the in vitro susceptibility test, and the patient was treated with amikacin, levofloxacin and ethambutol for 3 months. She recovered slowly, while some wrist swelling was still observed; however, pain and swelling significantly reduced. Eight months after anti-mycobacterial therapy, the patient showed complete recovery, and CRP, ESR, and WBC count were normal.

\section{Discussion and conclusion}

So far, M. jacuzzii has been only identified in patients with a history of breast surgery, involving implants from August to November 2003 at a single medical center in Tel Aviv, Israel. In this study, the prevalence of surgical site infection caused by $M$. jacuzzii following breast implantation was determined. The infectious agent was

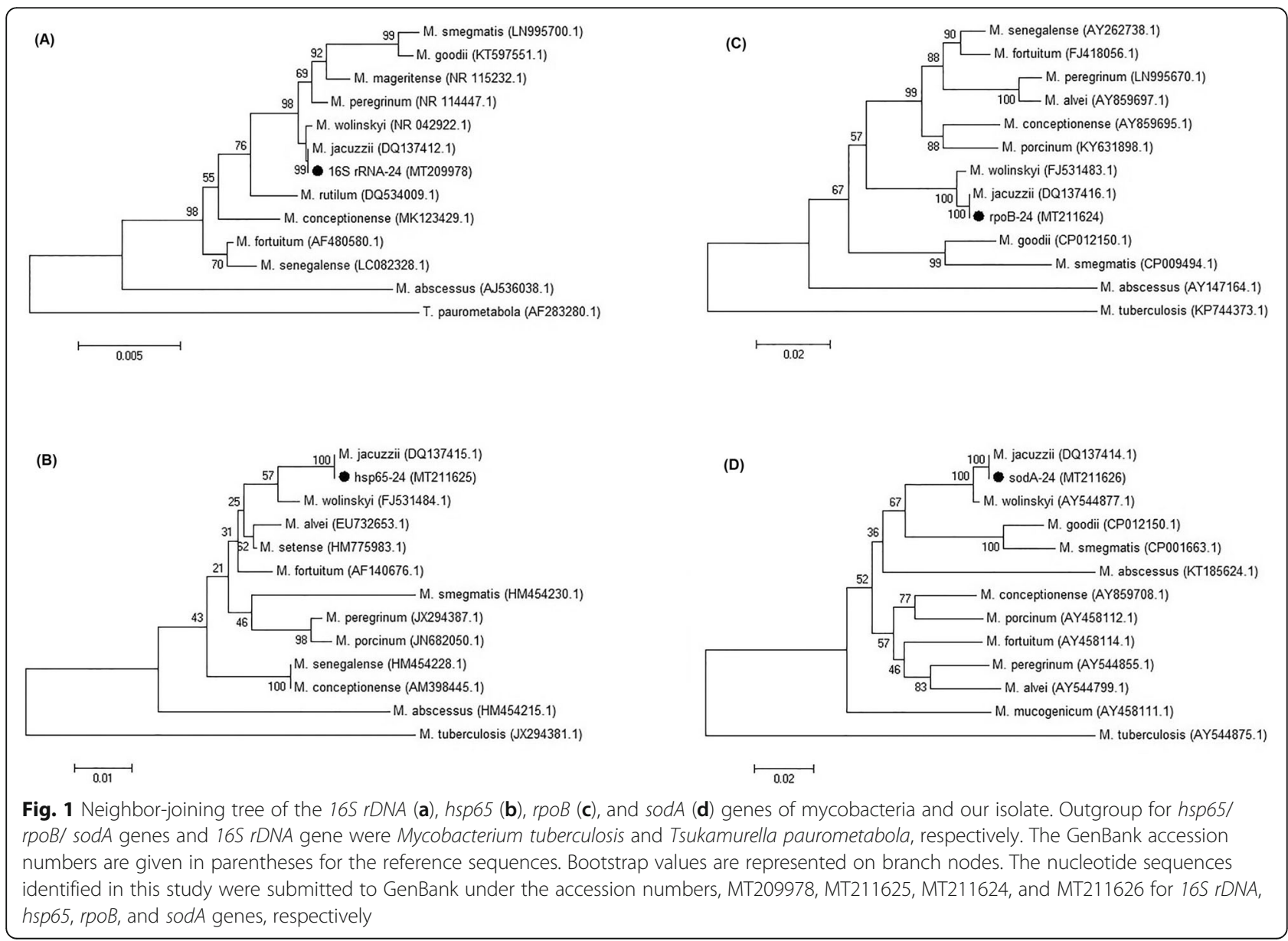


identified during surgery by an asymptomatic surgeon, who had collected the isolate from his whirlpool and suggested that this bacterium might be transmitted through human-to-human contact [5].

To the best of our knowledge, synovial infection caused by $M$. jacuzzii has not been previously reported. In this regard, Olsen et al. evaluated 66 cases of synovitis caused by NTM, including $M$. avium complex, $M$. terrae, M. nonchromogenicum, M. malmoense, M. haemophilum, and M. xenopi. The majority of these patients had an immunodeficiency and a history of wound, trauma, or invasive medical procedure around the joints [7]. The studied patient had received methylprednisolone, but she did not have any history of trauma or surgery on her wrist; therefore, we were unable to identify the entry route of the pathogen. However, this may not be an unusual occurrence, as in only $39 \%$ of mycobacterial synovitis cases, the source of inoculum can be identified for the infectious agent [7].

Mycobacterial synovitis therapy generally includes surgical excision and antibiotic administration [7]. However, due to the lack of clinical experience and underreporting of this isolate, the optimal treatment for $M$. jacuzzii infection has been standardized, including the duration of therapy, anti-mycobacterial drugs, and combination therapy. Only one study has explained the clinical course of M. jacuzzii infection. It was found that the removal of implants, total capsulectomy, and excision of all granulated tissue were effective treatments, but did not involve antimicrobial therapy [5].

Since only a limited number of anti-mycobacterial agents exhibit in vitro activity against $M$. jacuzzii, a combination therapy was initiated in our case with drugs to which the bacterium was susceptible, including amikacin, levofloxacin, and ethambutol. The outcomes of treatment after 8 months were positive, and no recurrence of infection was reported in the patient. In addition to other well-known NTM, our findings suggest that $M$. jacuzzii strain should be considered as a possible cause of synovitis.

\section{Abbreviations \\ M. jacuzzii: Mycobacterium jacuzzil; CRP: C-reactive protein; ESR: Erythrocyte sedimentation; WBC: White blood cells; NTM: Nontuberculous mycobacteria; AFB: Acid-fast bacillus; RGM: Rapidly-growing mycobacteria; DST: Drug susceptibility pattern; CLSI: Clinical and Laboratory Standards Institute}

\section{Acknowledgements}

We thank the patient for granting us permission to publish this information.

\section{Authors' contributions}

FS and MM: performed the experiments; FV and SDS: analyzed and interpreted data; AF: designed and supervised clinical study, interpreted data, read and approved manuscript. All authors read and approved the final manuscript.

\section{Funding}

None.

\section{Availability of data and materials}

All the data supporting the findings is contained within the manuscript. Sequence data of this organism that support the findings of this study have also been deposited in GenBank database with the accession number MT209978 for 165 rDNA gene (https://www.ncbi.n/m.nih.gov/nuccore/MT2 09978) and MT211624 (https://www.ncbi.nlm.nih.gov/nuccore/MT211624), MT211625 (https://www.ncbi.nlm.nih.gov/nuccore/MT211625), and MT211626 (https://www.ncbi.nlm.nih.gov/nuccore/MT211626) for rpoB, hsp65, and sodA genes, respectively.

\section{Ethics approval and consent to participate}

This study was in compliance with the 1975 Declaration of Helsinki and local regulations. It was also approved by the Ethics Committee of Pasteur Institute of Iran (IR.PII.REC.1394.54). Written informed consent was obtained from the patient.

\section{Consent for publication}

Patient's written informed consent to publish potentially identifying images and clinical details was obtained.

\section{Competing interests}

The authors declare no conflicts of interest.

Received: 11 July 2020 Accepted: 30 August 2020

Published online: 16 September 2020

References

1. Rivero-Lezcano OM, González-Cortés C, Mirsaeidi M. The unexplained increase of nontuberculous mycobacteriosis. Int J Mycobacteriol. 2019; $8(1): 1-6$.

2. WiMA. Treatment of Extrapulmonary Nontuberculous mycobacterial diseases. Infect Chemother. 2019;51(3):245-55.

3. Davari M, Irandoost M, Sakhaee F, et al. Genetic diversity and prevalence of nontuberculous mycobacteria isolated from clinical samples in Tehran, Iran. Microb Drug Resist. 2019;25(2):264-70.

4. Nour-Neamatollahie A, Ebrahimzadeh N, Siadat SD, et al. Distribution of non-tuberculosis mycobacteria strains from suspected tuberculosis patients by heat shock protein 65 PCR-RFLP. Saudi J Biol Sci. 2017;24(6):1380-6.

5. Rahav G, Pitlik S, Amitai Z, et al. An outbreak of Mycobacterium jacuzzii infection following insertion of breast implants. Clin Infect Dis. 2006; 43(7):823-30.

6. Woods G, Brown-Elliott A, Conville $P$, et al. Susceptibility testing of mycobacteria, nocardiae, and other aerobic actinomycetes. 2nd ed. Susceptibility Testing: Clinical and Laboratory Standards Institute (CLSI); 2011.

7. Olsen RJ, Cernoch PL, Land GA. Mycobacterial synovitis caused by slowgrowing nonchromogenic species: eighteen cases and a review of the literature. Arch Pathol Lab Med. 2006;130(6):783-91.

\section{Publisher's Note}

Springer Nature remains neutral with regard to jurisdictional claims in published maps and institutional affiliations. 